\title{
Androids: application of EAP as artificial muscles to entertainment industry
}

\author{
D. Hanson ${ }^{\mathrm{a} 1}$, G. Pioggia ${ }^{\mathrm{b} 2}$, Y. Bar Cohen ${ }^{\mathrm{c} 3}$, D. de Rossi ${ }^{\mathrm{b} 2}$ \\ ${ }^{a}$ Disney, ${ }^{b}$ U. Of Pisa, Italy, 'Jet Propulsion Lab, Pasadena, CA.
}

\begin{abstract}
The classic movie Metropolis (1926), which is nowadays considered a cinema milestone, has shown the possibility to build robots called androids that are science and fiction run together to realize a dream: the human-like robot. In that movie, Dr. Rotwang transforms a simple and cold calculating robot into the body of a beautiful woman. Robots have often been depicted as metal creatures with cold steel bodies, but there is no reason why metals should be the only kind of material for construction of robots. The authors examined the issues related to applying electroactive polymers materials (EAP) to the entertainment industry. EAP are offering attractive characteristics with the potential to produce more realistic models of living creatures at significantly lower cost. This paper seeks to elucidate how EAP might infiltrate and ultimately revolutionize entertainment, showing some applicative examples.
\end{abstract}

Keywords : Electroactive Polymers, EAP, Actuators, Entertainment, Animatronics, Android, Anthroids Humanoid, and Science Fiction.

\section{INTRODUCTION}

Current robots are constructed of metal or plastic frames, motors, gears, electronic circuit boards, and solid shapes that allow producing efficient machines but inefficient human characters, bodies and faces. In this design, the main structure of the robot is its exoskeleton. In many cases, a "skin" like material is placed over the exoskeleton but it is for appearance only. Exploiting the properties of electroactive polymers as artificial muscles may enable the movement of the covering skin, define the character and provide human-like expressivity. Such capabilities would offer great benefits to the entertainment industry. Portraying androids, animatronics and animals are essential to entertainment, and for some of the entertainment applications, long-term performance is not critical. Abundant movies use robotics and/or digital rendering to simulate organisms; examples include A Bug's Life, Deep Blue Sea, The Matrix, Mighty Joe Young, and many others. EAP could significantly enhance traditional character simulation technologies, adding biological accuracy to animatronics' actuation [Hanson and Pioggia, 2001].

In lab tests, EAP has already shown that it can match critical properties of biological muscle [Bar-Cohen, 1999, 2000, 2001a\&2001b, Zhang et al., 1999]. EAP also promises to be effective in configurations homologous to animal musculature - a strong advantage over other forms of actuation [Full and Meijer, 2001]. Although EAP has yet to demonstrate these capacities in character animation, recent materials breakthroughs bring the possibility tantalizingly close. EAP materials have characteristics attractive to the entertainment industry, offering the potential to more effectively model living creatures at significantly lower cost [Dickinson, et al, 2000]. Visco-elastic EAP materials could provide more lifelike aesthetics, vibration and shock dampening, and more flexible actuator configurations. Moreover, multiple studies have shown that the visco-elastic properties of animal tissue are key to locomotion and general stability. In these regards, EAP is the only actuation technology to date that earns the moniker "artificial muscle". To make it so, collaboration between academia and industry will be indispensable. If EAP proves itself in entertainment, it will find ample resources for further research and development.

\section{APPLICATION OF EAP TO ANDROIDS}

EAP-actuated animatronics may give birth to the darling of science fiction: the Android (or Anthroid, to be gender neutral). Such sentient beings designed to specification would be ideal for entertainment. EAP-based anthroids could star in movies, roam around theme parks and serve as tele-robots or interface devices for the Web. Given the likely moderate cost of EAP actuators, making anthroids that are driven by such actuators could quickly pervade our world as servants or even peers. Possibly, even props such as an anthroid's clothing could be animated using this type of actuation

\footnotetext{
${ }_{2}^{1}$ Walt Disney Imagineering, 1401 Flower Street, Glendale, CA 91221-5020,323-468-1133 dayofid@ hotmail.com

${ }_{3}^{2}$ Facolta di Ingegneria, Centro "E. PIAGGIO", Universita di Pisa, Via, Diotisalvi, 256100 Pisa, Italy

3 JPL/Caltech (MS 82-105), 4800 Oak Grove Drive, Pasadena, CA 91109-8099, yosi@ipl.nasa.gov, web: http://ndeaa.jpl.nasa.gov
} 
materials. With an estimated two billion dollars per year spent on character animation, the entertainment industry may be the most salient vehicle for the development of EAP-based humanoid robotics. Key to tapping these resources, however, is a first-generation, functional product. Devising inexpensive and robust EAP-based animation that outperforms rival entertainment technology is the task at hand.

Recently it has been shown that EAPs can exert significant displacements and reasonable forces offering many improvements over traditional actuation technologies, both in function and form. Functionally, EAP may have higher power densities than other type of actuators. Being electric, EAP actuators would do away with bulky and fault-prone hydraulic actuators, respond faster and eradicate the mess associated with hydraulic failure. Given the generally moderate cost of polymers, such actuators are likely to be more affordable than their competing alternatives. EAP actuators' unitary mechanical form allows them to be packed into smaller volumes. Volume is further conserved by EAP's flexibility and potentially moldable nature. Such flexibility expands the options for mechanical configuration of actuators. Absence of gearing and transmission reduces points of possible failure. EAP can act as dampener, insulating a mechanism from shock and damaging resonant vibrations. Elastomeric qualities will allow for spring-mass energy cycling, improved efficiency and stability in locomotion and gesture, and a generally more lifelike appearance [Amos, et al. 1996; and Bailey, et al. 2000].

Introducing new technology to an existing field requires addressing technical challenges while providing results unobtainable by conventional means. In doing so, the technology needs to be reliable and affordable. Should EAP succeed in entertainment, it will find many uses aside from obvious narrative applications. EAP may also unleash humanoid devices, telepresence tourism, and athletics-enhancement devices (a.k.a. flapping Icarus wings or gargantuan Hercules arms). A more immediate use of EAP could be in theme park ride seats, leading to a more "gripping" sensory experience. As the craze over Sony's Aibo demonstrates, there is a huge demand for ambulating character products. Walking and running figures require the same basic characteristics as for gestures - dynamic stability is paramount [Pratt, 2000]. While the science behind dynamic ambulation as a whole is still emerging, the dynamics of sprawled animal locomotion (lizards, cockroaches, etc.) are becoming more completely understood. Sprawled posture would be the most pproachable form for EAP mobility in entertainment. Although no autonomous, dynamic biped yet exists, much headway is being made into engineering bipedal dynamic locomotion. Bipeds would be the more ambitious arena for EAP actuator exploration, but ultimately more rewarding for character animatronics. The key to making these possibilities a reality is to overcome the challenges that are involved while harnessing the capabilities.

\section{REQUIREMENTS FOR EAP APPLICABILITY TO ENTERTAINMENT}

Preferred characteristics of EAP actuators include high strain, high power density, robustness and durability, and the support of fully developed adjunct technology (fasteners, drivers etc.). Additionally, the following specifications would need to be met for EAP actuators to be reasonable for entertainment use:

- For movie use, longevity should be in the thousands of cycles, whereas, for theme park use, hundreds of thousands of cycles.

- Force generation needs to be at least 15 times weight of actuator, and up to 200 grams or better.

- Response time should be in the low milliseconds. The speed of actuation needs to be at least 5-cm/sec

- Displacement should be at least $10 \%$ of the length of the actuator.

- The actuators would preferably be configurable in sheets or bundles.

- End fasteners should be integrated into the actuators.

- The actuators should be dry, and if not, they should be thoroughly sealed.

- They should be inexpensive (less than $\$ 300$ for a midsize $510 \mathrm{~cm}$ (2-4 inch) actuator with driver). They also need to be easy to handle and use.

- For facial animation, the most useful actuators would be 2.5 to $7.5 \mathrm{~cm}$ (1" to 3") long actuators with $20 \%$ strain or displacement, weighing a maximum 15 grams.

\section{AVAILABLE EAP MATERIALS}

Since the early 1990s, several EAPs have emerged that exhibit significant electromechanical response [Bar-Cohen, 1999, 2000, 2001a\&2001b, Zhang et al., 1999]. To assist potential users of EAPs in assessing the applicability of the various materials, the Bar-Cohen [2001] divided them into two major groups: ionic and electronic, based on their actuation mechanism. Examples of the currently available materials in each of these two groups are listed in Table 1. The general advantages and disadvantages of each these two groups are listed in Table 2. The capability of these EAP materials to generate a large stain cannot be matched by any alternative electroactive material including piezoceramics and shape memory alloys. However, most of these materials are still in their emerging stage and further development is needed $\mathbf{b}$ address the challenges to their practical application. However, no existing actuation technology has presented so many potential benefits to animatronic character animation as EAP, and applying them with principles of dynamic stability can 
make all the difference [Brooks, 1991; McComb, 2000; Menzel and D'Aluisio, 2000; and Scharft, 2000]. If EAP actuation proves practical, it will certainly revolutionize animatronics.

TABLE 1: Example of EAP materials

\begin{tabular}{|ll|ll|}
\hline \multicolumn{2}{|c|}{ Electronic EAP } & & \multicolumn{1}{c|}{ Ionic EAP } \\
\hline$\bullet$ & Dielectric EAP & $\bullet$ & Carbon Nanotubes (CNT) \\
$\bullet$ & Electrostrictive Graft Elastomers & $\bullet$ & Conductive Polymers (CP) \\
$\bullet$ & Electrostrictive Paper & $\bullet$ & ElectroRheological Fluids (ERF) \\
$\bullet$ & Electro-Viscoelastic Elastomers & $\bullet$ & Ionic Polymer Gels (IPG) \\
- & Liquid Crystal Elastomers (LCE) & $\bullet$ & Ionic Polymer Metallic Composite (IPMC) \\
$\bullet$ & Ferroelectric Polymers & & \\
\hline
\end{tabular}

TABLE 2: A Summary of the advantages and disadvantages of the two basic EAP groups

\begin{tabular}{|c|c|c|}
\hline $\begin{array}{l}\text { EAP type } \\
\text { Electronic } \\
\text { EAP }\end{array}$ & \begin{tabular}{l}
\multicolumn{1}{c}{ Advantages } \\
- $\quad$ Long life in room conditions \\
- $\quad$ Rapid response (mSec levels) \\
- Can hold strain under DC activation \\
- Induces relatively large actuation \\
forces
\end{tabular} & \begin{tabular}{l}
\multicolumn{1}{c}{ Disadvantages } \\
- Requires high voltages $(\sim 150 \mathrm{MV} / \mathrm{m})$ \\
- Requires compromise between strain and stress \\
- Glass transition temperature is inadequate for low \\
temperature actuation tasks
\end{tabular} \\
\hline Ionic EAP & $\begin{array}{l}\text { - Large bending displacements } \\
\text { - Provides mostly bending actuation } \\
\text { (longitudinal mechanisms can be } \\
\text { constructed) } \\
\text { - Requires low voltage }\end{array}$ & $\begin{array}{l}\text { - Except for CPs, ionic EAPs do not hold strain under } \\
\text { DC voltage } \\
\text { - Slow response (fraction of a second) } \\
\text { - Bending EAPs induce a relatively low actuation force } \\
\text { - Except for CPs, it is difficult to produce a consistent } \\
\text { material (particularly IPMC) } \\
\text { - In aqueous systems the material sustains hydrolysis at } \\
>1.23-\mathrm{V}\end{array}$ \\
\hline
\end{tabular}

\section{ANDROID FACIAL EXPRESSIONS}

EAP actuation should allow more physiologically accurate animatronic design making movement of the resulting animation to look more inherently organic [Pollack, et al. 2001]. A near term objective for the development of EAP for animatronics can be the actuation of various elements of the android or a toy face to make facial expressions [Darvin, 1872; Lamb, et al. 1999]. The softness and elasticity of such actuators can be foreseen to eliminate the distracting surface-distortion caused by rigid actuator elements. Their compliance and dampening characteristics closely correspond with those of biological muscle, simplifying controls and leading to more gesturally accurate animation. Such actuators can be made either flat or bundled, like real muscle and they can be imbedded directly into rubber skins that are currently in widespread use for special effects. By integrating artificial muscles into layers, the complex musculature of the human face could be emulated. Such a complex array of actuators will likely render much more subtle and intricate expressions than contemporary facial anima tronics.

Development of an anthroid face using EAP actuators is, arguably, one of the most important step in the introducing EAP technology to character animatronics [Hanson and Pioggia, 2001; and Pioggia et al. 2001]. Currently the University of Pisa, Italy, is emulating facial anatomy with a robotic humanoid head, using dielectric elastomer, so called electrostatically stricted polymer (ESSP) muscles (Figures 1 and 2). This project is seeking insights into humanity's primary communicative bodily system: the countenance. The face is the most captivating and hypnotic organ. The mere sight of facial expressions tends to evoke uncontrollable surges of emotion, and conversely, one's face will often betray thoughts and emotions hidden to oneself. Mimicking the human face with a simplified array of electroactive artificial muscles may improve relations between humans and machines.

FIGURE 1: The Android face of the University of Pisa.

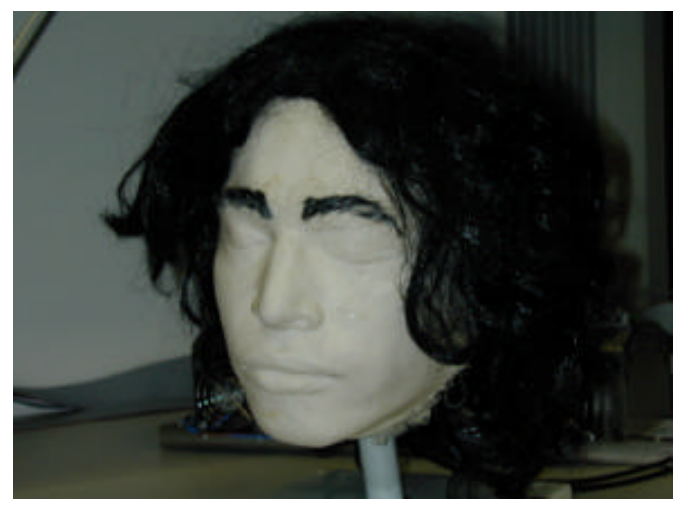


Human facial expressions involve complex characteristics, where wrinkles lie dormant all over the face, invisible until an expression is enacted. Opposing movements allows evoking radically different types of folding and bunching of skin. Human skin is extremely soft, and is intimately affected by the pulling of various internal layers of tissues. Since human skin is an elaborate web of liquid saturated cellular tissue, it is tricky to emulate with solid elastomer. Patient trial and error may be our best hope to isolate an effective multi-modal facial skin simulation and soft-tissue actuators are highly desirable. Gels might be more effective than other elastomers at simulating tissues, particularly for the various origami-like folding configurations typical to human skin. A possible engineering approach could be the use of a gel saturated into a tissue matrix using materials with properties and structures similar to biological tissues and fluids on a sub-millimeter scale. Biotechnology might have useful inroads cut into this field. There are many robotics researchers investigating the emulation of human expression and cross-pollination with the entertainment industry would behoove both groups.

From a distance, body gesture is all that reads of a character's movement. Our visual intuition is keenly sensitive to the appearance of weight and balance; we just know if something is off. Our natural gestures include compliance (springiness), yet our movements are precise. One reason for this is that we have legion actuators and sensors, and distributed controls. If EAP become robust, inexpensive, small, and mass-produced, then perhaps arrays of thousands of sensors and actuators could be deposited in a robot's simulated flesh. In the meantime, mathematical principles derived from biology could allow us to approximate balance, for much improved visual effect and heightened precision [Full, 2001]. The development goal could be to compile a database of gestures and expressions, with dynamic mechanical analysis, physiological analysis (part and parcel of the latter), and psychological analysis. Creating an automated codex for inputting and analyzing expressions, gestures, and various coordinated movements would be useful for this database.

FIGURE 2: Construction of the android head (see Figure 1) and various facial expressions.

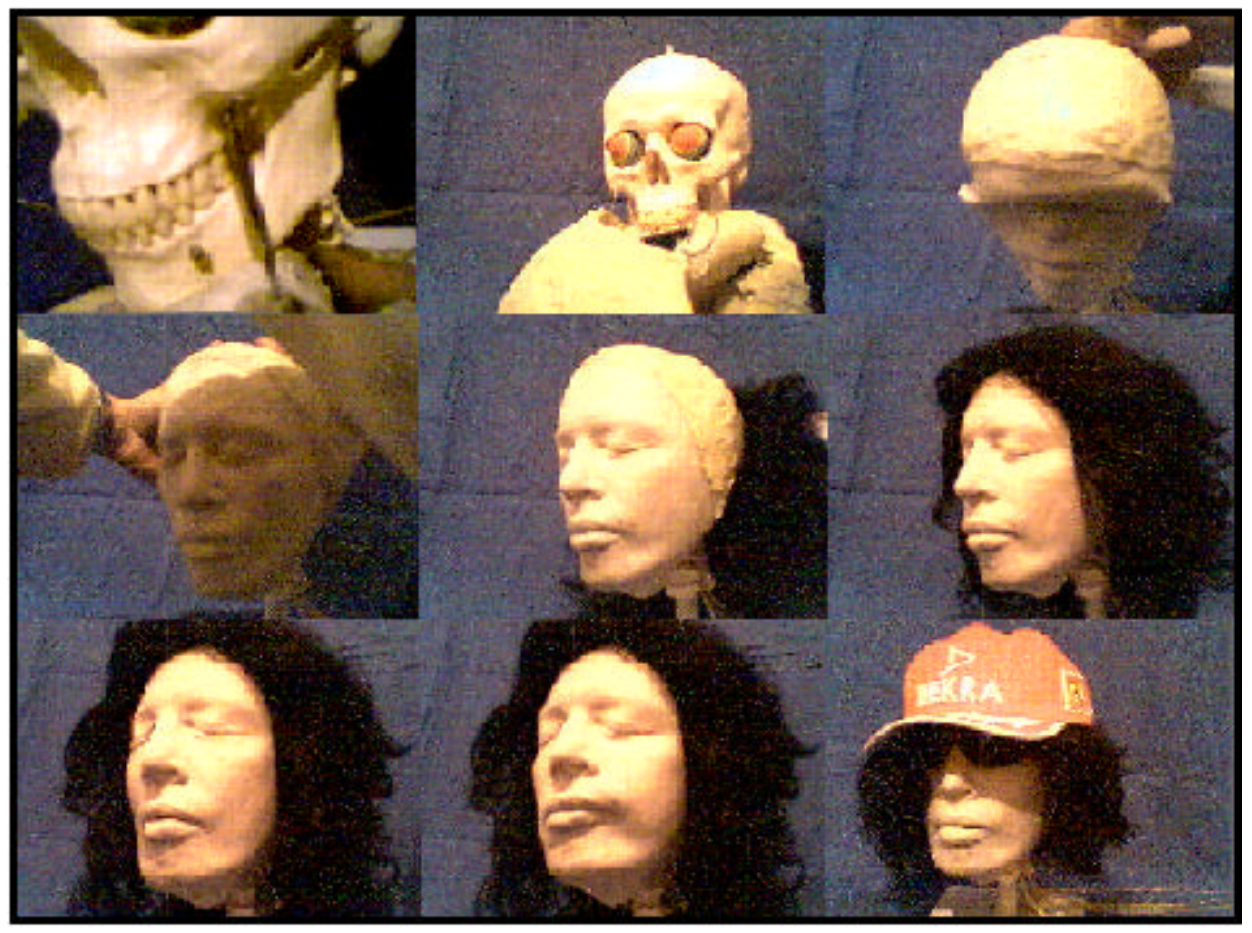

CONCLUSION

EAP may change animatronics, but much effort will be required. In addition to developing better EAP actuation, a discipline of visco-elastic engineering will need to supplant the traditional engineering of rigid structures including controls strategies. If one single effective EAP actuator product is introduced to the entertainment market, it could generate a flurry of resources for further research and development. To be certain, symbiosis between academia and the animatronics community is crucial at this stage to tune EAP towards entertainment needs. Many other technologies also support the use of EAP in entertainment. Of particular interest are Artificial Intelligence to imbue the animatronics with life, and Shape Deposition Manufacturing, allowing rapid prototyping of visco-elastic mechanisms with imbedded actuators. Successful EAP animatronics will require vigorous study of human physiology and physical systems, to derive applicable engineering principles. A healthy start has been made in an EAP actuated anthroid head, as reported herein, however much work must be done before this system can be useful to entertainment. Though many obstacles lie in the way of useful EAP actuation for entertainment, overcoming the challenges would benefit numerous other fields. The EAP technology represents many advantages over traditional actuation, and thus it easily warrants the adjective "revolutionary". 


\section{REFERENCES}

Amon C.H., J.L. Beuth, R. Merz, F.B. Prinz, L.E. Weiss, "Shape Deposition Manufacturing with Microcasting: Processing, Thermal and Mechanical Issues", Journal of Manufacturing Science and Engineering, (1996).

Bailey S. A., J.G. Cham, M. R. Cutkosky, and R.J. Full. "Biomimetic Robotic Mechanisms via Shape Deposition Manufacturing," Robotics Research: the Ninth International Symposium, J. Hollerbach and D. Koditschek (Eds), Springer-Verlag, London, (2000) pp. 403-410.

Bar-Cohen Y. (Ed.), "Electroactive Polymer (EAP) Actuators as Artificial Muscles - Reality, Potential and Challenges," SPIE Press, (In press, 2001a).

Bar-Cohen Y., "EAP Applications, Potentials and Challenges," Chapter 21, Topic 8, (2001b) ibid.

Bar-Cohen Y., (Ed.), Proceedings of the SPIE's Electroactive Polymer Actuators and Devices Conf., 7 th Smart Structures and Materials Symposium, Vol. 3987, ISBN 0-8194-3605-4 (2000) pp. 1-360.

Bar-Cohen Y., (Ed.), Proceedings of the SPIE's Electroactive Polymer Actuators and Devices Conf., 6 th Smart Structures and Materials Symposium, Volume 3669, ISBN 0-8194-3143-5, (1999), pp. 1-414.

Brooks RA, "Intelligence without Reason,” MIT AI Lab Internal Report, (1991).

Darwin, C., Ekman, P. (Ed.), The Expression of the Emotions in Man and Animals, Oxford University Press, New York, (1998/1872).

Dickinson M.H., Farley, C.T., Full, R.J., Koehl, M. A. R., Kram R., and Lehman, S., "How animals move: An integrative view," Science 288, (2000), pp. 100-106.

Full R. J., and K, Meijer, "Natural Muscles as an Electromechanical System," Chapter 3, Topic 2, Electroactive Polymer (EAP) Actuators as Artificial Muscles - Reality. Potential and Challenges, Bar-Cohen Y. (Ed.), SPIE Press, (in press, 2001), pp. 67-83.

Hanson D., and G. Pioggia, "Applications of EAP to Entertainment Industry," Chapter 18, Topic 7, Electroactive Polymer (EAP) Actuators as Artificial Muscles - Reality. Potential and Challenges, Bar-Cohen Y. (Ed.), SPIE Press, (in press, 2001), pp. 535-565

Lamb S. M., "Pathways of the Brain," The Neurocognitive Basis of Language, Amsterdam \& Philadelphia: John Benjamins Publishing Co., (1999).

McComb G., The Robot builder's bonanza, Second Edition, McGraw Hill (2000)

Menzel P., F. D’Aluisio, Robo sapiens: Evolution of a New Species, Boston, MIT Press, (2000).

Pioggia G., Di Francesco, F., Chiarelli, P., De Rossi, D., "An human-like android face equipped with EAP artificial muscles to endow expressivity" Proceedings of the SPIE's Electroactive Polymer Actuators and Devices Conf., 8 th Smart Structures and Materials Symposium, (2001) (to be published).

Pollack G. H., F. A. Blyakhman, F. B. Reitz, O. V. Yakovenko, and D. L. Dunaway, "Natural Muscles as a Biological system," Chapter 2, Topic 2, Electroactive Polymer (EAP) Actuators as Artificial Muscles - Reality, Potential and Challenges, Bar-Cohen Y. (Ed.), SPIE Press, (in press, 2001), 47-66.

Pratt J., "Exploiting Inherent Robustness and Natural Dynamics in the Control of Bipedal Walking Robots", Ph.D. thesis, Computer Science Department, Massachusetts Institute of Technology, Cambridge, Massachusetts, (2000).

Schraft R.D., and G. Schrmierer, Service Robots, A K Peters, Ltd., Natick, MA, ISBN 1-56881-109-8 (2000), pp.145153.

Zhang Q.M., T. Furukawa, Y. Bar-Cohen, and J. Scheinbeim, Electroactive Polymers (EAP)," ISBN 1-55899-508-0, MRS Symposium Proceedings, Vol. 600, Warrendale, PA, (1999), pp 1-336. 